\title{
Étude numérique
}

\section{des régimes transitoires dans les canaux}

\section{Numerical study of transient conditions in canals}

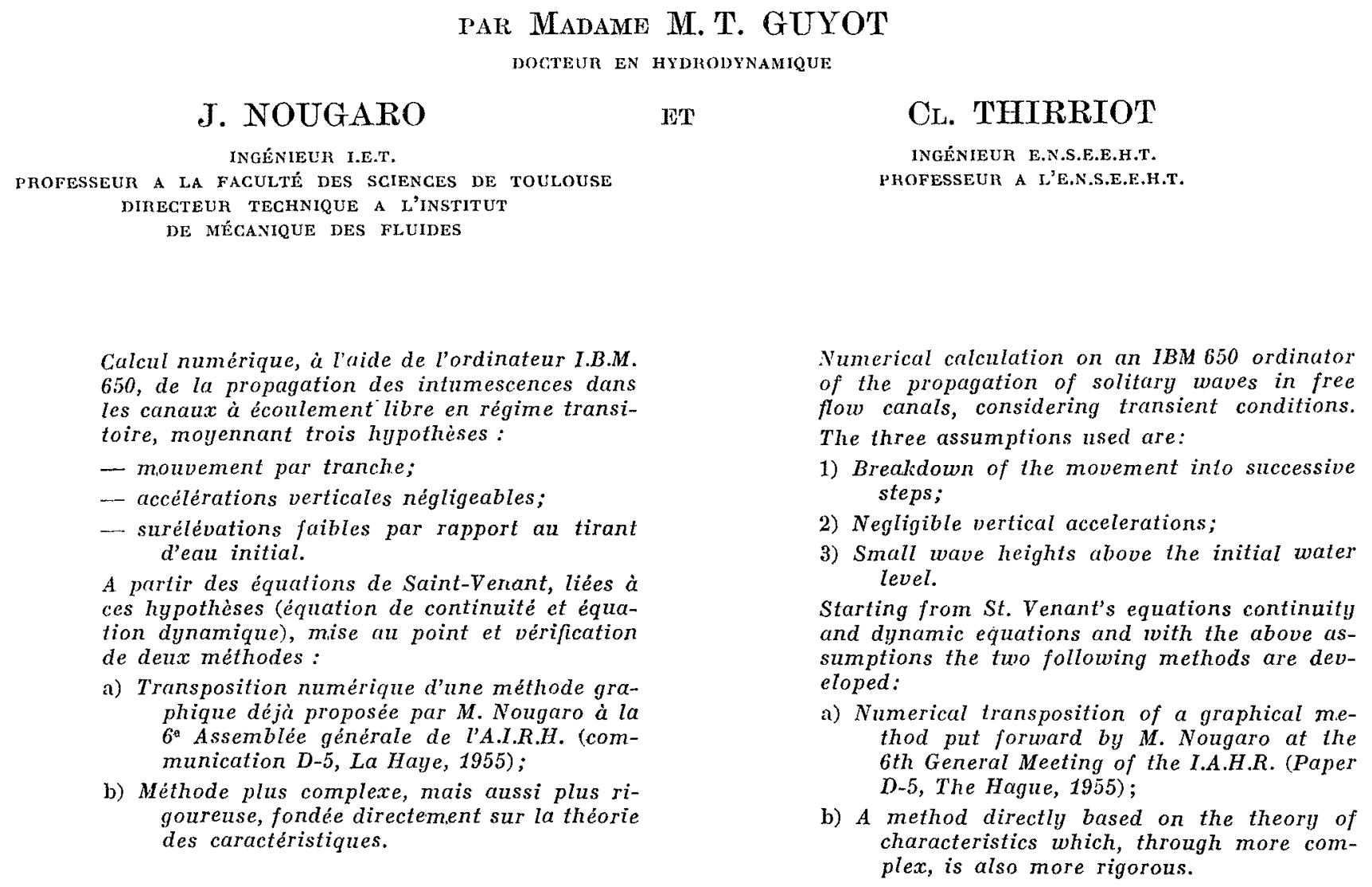

\section{INTRODUCTION}

Comme dans beaucoup d'autres domaines de la science et de la technique, les machines à calculer modernes peuvent jouer un rôle important dans l'étude des phénomènes transitoires hydrauliques et particulièrement dans l'étude de la propagation des intumescences.

Dès 1958, le Laboratoire d'Hydraulique de Toulouse a mis au point un premier programme de calcul numérique de la propagation d'intumescences.
Depuis cette date, plusieurs autres programmes ont été établis donnant à la méthode plus de souplesse et de généralité.

C'est ainsi que nous présentons, dans cette communication, notre contribution à l'étude numérique des régimes transitoires dans les canaux d'usines.

Rappelons brièvement les hypothèses que l'on adopte habituellement dans l'étude théorique des intumescences : 
- le mouvement de l'eau se fait par tranche,

- les accélérations verticales sont négligeables,

- les surélévations sont faibles par rapport au tirant d'eau initial.

Moyennant ces hypothèses, on aboutit au systèmes d'équations établies par Saint-Venant, l'équation de continuité :

$$
\frac{\partial \Sigma}{\partial t}+\Sigma \frac{\partial v}{\partial x}+v \frac{\partial \Sigma}{\partial x}=0
$$

et l'équation dynamique :

$$
\frac{\partial v}{\partial t}+v \frac{\partial v}{\partial x}+k v^{2}-g i-\frac{\partial h}{\partial x}=0
$$

oủ $\Sigma$ est la section mouillée;

$v$ la vitesse moyenne d'une tranche liquide;

$k$ le coefficient de perte de charge;

$i$ la pente du radier;

$q_{\rho}$ le débit latéral par unité de longueur.

A partir de ces équations, nous avons mis au point et vérifié deux méthodes de calcul numérique de la propagation d'intumescence.

L'une, méthode très simple, a été la transposition numérique d'une méthode graphique proposée par l'un de nous (1).

La seconde, méthode plus complexe, mais aussi plus rigoureuse, est fondée directement sur la théorie des caractéristiques.

Les calculs ont été effectués à l'aide de l'ordinateur I.B.M. 650 de l'Institut de Calcul numérique de la Faculté des Sciences de Toulouse.

\section{I. - PRINCIPE DE LA MÉTHODE}

Les équations fondamentales précitées établies dans le cas d'un canal s'écrivent:

$$
\begin{gathered}
\frac{\partial v}{\partial t}+v \frac{\partial v}{\partial x}+k v^{2}=g\left(i-\frac{\partial h}{\partial x}\right) \\
\frac{\partial v}{\partial x}+\frac{v}{\Sigma} \frac{\partial \Sigma}{\partial x}+\frac{1}{\Sigma} \frac{\partial \Sigma}{\partial t}=\frac{q_{l}}{\Sigma}
\end{gathered}
$$

Généralement, les intumescences sont engendrées par des discontinuités de fonctionnement (variation brusque de débit pour manœuvre brusque ou de la dérivée du débit pour une manouvre linéaire).

Ces discontinuités se propagent et leur loi de déplacement est représentée, dans le plan $(x, t)$ par exemple, par des lignes que l'on qualifie de caractéristiques.

La détermination de ces lignes caractéristiques peut être obtenue à partir des équations fondamentales en remarquant que, par définition, ces lignes sont lieu de discontinuité et par conséquent, sur de telles lignes, les dérivées partielles ne sauraient admettre une valeur unique.

Cette propriété a été signalée par Monge et utilisée par Massau.

Les dérivees partielles vérifient aussi les relations :

$$
\begin{gathered}
d v=\frac{\partial v}{\partial t} d t+\frac{\partial v}{\partial x} d x \\
d \Sigma=\frac{\partial \Sigma}{\partial t} d t+\frac{\partial \Sigma}{\partial x} d x
\end{gathered}
$$

Les équations (1), (2), (3), (4) peuvent s'écrire, sous forme matricielle, qui permettra la détermination des inconnues $\partial v / \partial t, \partial v / \partial x, \partial \Sigma / \partial l$, $\partial \Sigma / \partial x$, après avoir exprimé $\partial h / \partial x$ en fonction de $\partial \Sigma / \partial x$.

EXPRESSION DE $\partial h / \partial x$ :

Canal cylindrique. - Pour un profil déterminé, $h$ est une fonction connue plus ou moins compliquée de $\Sigma$; donc $\partial h / \partial x$ est déterminé en fonction de $\partial \Sigma / \partial x$.

Par exemple, on peut poser :

$$
\delta \Sigma_{x}=\mathrm{B} \delta h_{x}
$$

B étant la largeur au miroir, d'où :

$$
\frac{\partial h}{\partial x}=-\frac{1}{B} \frac{\partial \Sigma}{\partial x}
$$

Ouvrage non cylindrique. - La relation entre $\Sigma$ et $h$ varie longitudinalement. Soit un coefficient de forme qui tient compte de la modification longitudinale du profil, tel que $\Sigma=f(h, \alpha)$.

Le coefficient de forme a peut englober plusieurs paramètres.

(1) J. Nougaro. Note à I'A.I.R.H. Méthode graphique pour le calcul de la propagation des intumescences dans les canaux découverts.

Compte rendu de la $6^{\circ}$ Assemblée Générale de l'A.I.R.H. (communication D 5, La Haye, 1955). 
Dans le cas d'une galerie conique à profil circulaire, $\alpha$ sera le diamètre $\varnothing$ de la section.

Dans l'hypothèse d'une section trapézoïdale, $\alpha$ désignera la largeur du radier $B_{0}$ et le fruit des berges $m$.

Dans les deux cas, la loi $\alpha(x)$ est connue.

Comment exprimer alors $\partial h / \partial x$ ?

$\Sigma$ dépend de $h$ et de $\alpha$, donc :

$$
\frac{\partial \Sigma}{\partial x}=\left(\frac{\partial \Sigma}{\partial h}\right)_{n=c t} \frac{\partial h}{\partial x}+\left(\frac{\partial \Sigma}{\partial x}\right)_{h=c t} \frac{\partial \alpha}{\partial x}
$$

$(\partial \Sigma / \partial h)_{a=c h}=\mathrm{B}$, car $\alpha=$ coustant est le cas d'un canal prismatique.

$\partial \alpha / \partial x$ est connu en fonction de $x$.

$(\partial \Sigma / \partial \alpha)_{l=c t}$ en un point $(x, \Sigma)$ est une fonction connue.

$\partial \Sigma / \partial x$ est la fonction inconnue principale, donc :

$$
\frac{\partial h}{\partial x}=\frac{1}{B}\left[\frac{\partial \Sigma}{\partial x}-\left(\frac{\partial \Sigma}{\partial \alpha}\right)_{h=c t} \frac{\partial \alpha}{\partial x}\right]
$$

En utilisant ce résultat, l'équation dynamique devient :

$$
\begin{aligned}
\frac{\partial v}{\partial t}+v \frac{\partial v}{\partial x} & +\frac{g}{\mathrm{~B}} \frac{\partial \Sigma}{\partial x} \\
& =g(i-j)+g \frac{1}{\mathrm{~B}}\left(\frac{\partial \Sigma}{\partial \alpha}\right)_{h=c t} \frac{\partial \alpha}{\partial x}
\end{aligned}
$$

Le terme $(1 / B)(\partial \Sigma / \partial \alpha) .(\partial \alpha / \partial x)$ est sans dimension, analogue aux pentes $i$ et $j$; nous le désignerons par $l$.

Pour simplifier les écritures, on peut poser :

$$
J=i-j+l \text {. }
$$

Ainsi l'écquation (1) s'ćcrit :

$$
\frac{\partial v}{\partial t}+v \frac{\partial v}{\partial x}+\frac{g}{B} \frac{\partial \Sigma}{\partial x}=g J
$$

Dans de nombreux cas, une condition aux limites impose une loi de débit. La plus simple est la constance du débit consécutive à une manœuvre brusque.

Pour simplifier le calcul à une limite, il y a intèrêt à prendre comme fonctions inconnues le débit $q$ et la section $\Sigma$.

Les équations fondamentales deviennent alors, en utilisant la transformation $v=(q / \Sigma)$ :

$$
\begin{gathered}
\Sigma \frac{\partial q}{\partial t}+q \frac{\partial q}{\partial x}-q \frac{\partial \Sigma}{\partial t}-\frac{q^{2}}{\Sigma} \frac{\partial \Sigma}{\partial x}+k q^{2} \\
=g \Sigma^{2}\left[i-\frac{\partial h}{\partial x}\right] \\
\frac{\partial q}{\partial x}+\frac{\partial \Sigma}{\partial t}=q_{l}
\end{gathered}
$$

qui, jointes à l'expression des différentielles $d q$ et $d \Sigma$, donnent en écriture matricielle :

$\left|\begin{array}{ccccc}\Sigma & q & -q & g \frac{\Sigma^{2}}{\mathrm{~B}}-\frac{q^{2}}{\Sigma} \\ 0 & 1 & 1 & 0 \\ d t & d x & 0 & 0 \\ 0 & 0 & d t & d x\end{array}\right| \times\left|\begin{array}{c}\frac{\partial q}{\partial t} \\ \frac{\partial q}{d x} \\ \frac{\partial \Sigma}{\partial t} \\ \frac{d \Sigma}{d x}\end{array}\right|=\left|\begin{array}{c}g \mathrm{~J}^{2} \\ \Sigma_{l} \\ d q \\ d \Sigma\end{array}\right|$

Nous trouvons la relation différentielle vérifiée par les lignes caractéristiques en annulant le déterminant caractéristique :

$$
\begin{aligned}
& r t^{2}\left(g \frac{\Sigma^{2}}{\mathrm{~B}}-\frac{q^{2}}{\Sigma}\right)+2 q d t . d x-\Sigma d x^{2}=0 \\
& \frac{d x}{d t}=\frac{q \pm \sqrt{g\left(\Sigma^{3} / \mathrm{B}\right)}}{\Sigma}=\frac{q}{\Sigma} \pm \sqrt{g(\Sigma / \mathrm{B})}
\end{aligned}
$$

ou en désignant, comme il est habituel, par $c$ la valeur de la célérité $\sqrt{g(\Sigma / \mathrm{B})}$ :

$$
d x / d t=v \pm c
$$

De même, nous trouvons la loi de fonctionnement sur les caractéristiques, en annulant le déterminant obtenu en remplaçant la dernière colonne du déterminant précédent par la colonne du deuxième membre.

$$
\left|\begin{array}{cccc}
\Sigma & q & -q & g \mathrm{~J} \Sigma^{2} \\
0 & 1 & 1 & q_{l} \\
d t & d x & 0 & d q \\
0 & 0 & d t & d \Sigma
\end{array}\right|=0
$$

Nous obtenons :

$$
\begin{aligned}
-\Sigma d q- & \frac{d x}{d t} \Sigma d \Sigma+2 q d \Sigma \\
& +d t\left[g \mathrm{~J} \Sigma^{2}+\eta_{l} \Sigma\left(\frac{d x}{d t}-\frac{q}{\Sigma}\right)\right]=0
\end{aligned}
$$

ou en remplaçant $d x / d t$ par sa valeur le long des lignnes caractéristiques :

$$
\begin{aligned}
-\Sigma d q-\left(\frac{q}{\Sigma} \pm c\right) \Sigma d \Sigma & +2 q d \Sigma \\
& +d t\left[g \Sigma^{2} \mathrm{~J} \pm c q_{l} \Sigma\right]=0
\end{aligned}
$$

soit :

$$
(v \mp c) d \beth-d q+d t\left[g \mathrm{~J} \Sigma \pm c q_{l}\right]=0
$$

Si on néglige le terme $g \mathrm{~J} \Sigma \pm c q_{l}$, nous avons, 
le long des caractéristiques, l'équation différentielle :

$$
d q=(v \mp c) d \Sigma
$$

\section{Remarques :}

1. Le signe devant le radical (ou le signe devant c) est différent pour une même caractérislique dans la valeur de la pente $d x / d t$ et dans l'expression de la pente $d q / d \Sigma$ :

$$
\frac{d x}{d t}=v \pm c \quad \frac{d q}{d \Sigma}=v=c
$$

Nous appellerons caractéristiques descendantes celles qui vérifient :

$$
\frac{d x}{d t}=v+c \quad \frac{d q}{d \Sigma}=v-c
$$

el caractéristiques montantes, celles qui vérifient :

$$
\frac{d x}{d t}=v-c \quad \frac{d q}{d \Sigma}=v+c
$$

Il est évident que les caractéristiques descendantes suivent le courant et que les caractéristiques montantes vont contre le courant.

Dans ce qui suit, nous n'étudierons que les cas où $v<c$, c'est-à-dire le régime fluvial, circonstance oil les caractéristiques ont des pentes de signes différents.

2. Les valeurs des pentes des caractéristiques sont les mêmes que celles définies et employées par l'un de nous (2).

3. La considération d'un canal dont la section varie de forme longitudinalement ou de l'influence d'un débit latéral n'offrirait aucune difficulté théorique.

4. La même méthode est applicable pour: l'étude des coups de bélier. Elle est utilisable dans la résolution de tous les systèmes d'équation aux dérivées partielles du premier ordre du type hyperbolique.

\section{II. - BASE DE LA MÉTHODE NUMÉRIQUE}

Le calcul numérique sera la traduction, en termes de différences finies, des résultats de la théorie des caractéristiques. Mais il n'est pas inutile le concrétiser les équations obtenues par une représentation graphique.

Considérons deux plans de coordonnées (fig. 1):

-.. un plan $x, t$ : espace-temps,

-- un plan $q, \Sigma$ : débit-section.

Un point de fonctionnement serait ainsi défini par quatre valeurs, $x$ fixant la position de la section, $t$ donnant la date, $q$ le débit et $\Sigma$ la section mouillée et par là, le tirant d'eau dans la section considérée à la date $t$.

Supposons connus deux points, $M$ et $N$, dans le plan $x$, $t$, et leurs homologues $M^{\prime}$ et $N^{\prime}$ dans le plan $q, \Sigma$.

A partir de $M$ et $M^{\prime}$, tracons les caractéristiques $\%$ et, à partir de $\mathrm{N}$ et $\mathrm{N}^{\prime}$, les caractéristiques $\beta$ dont la pente a un signe différent. Nous obtenons par intersection le point $P, P^{\prime}$.

Pour la mise en évidence du processus, supposons que les tronçons $\overline{\mathrm{MP}}, \overline{\mathrm{NP}}$ dans le plan $x$, $t$, et $\overline{\mathrm{N}}^{\prime} \overline{\mathrm{P}}^{\prime}$ et $\overline{\mathrm{M}}^{\prime} \overline{\mathrm{P}}^{\prime}$ dans le plan $(q, \Sigma)$ sont rectilignes.

(2) J. Nougaro, dans son étude de la propagation des intumescences par la méthode graphique des caractéristiques courbes.
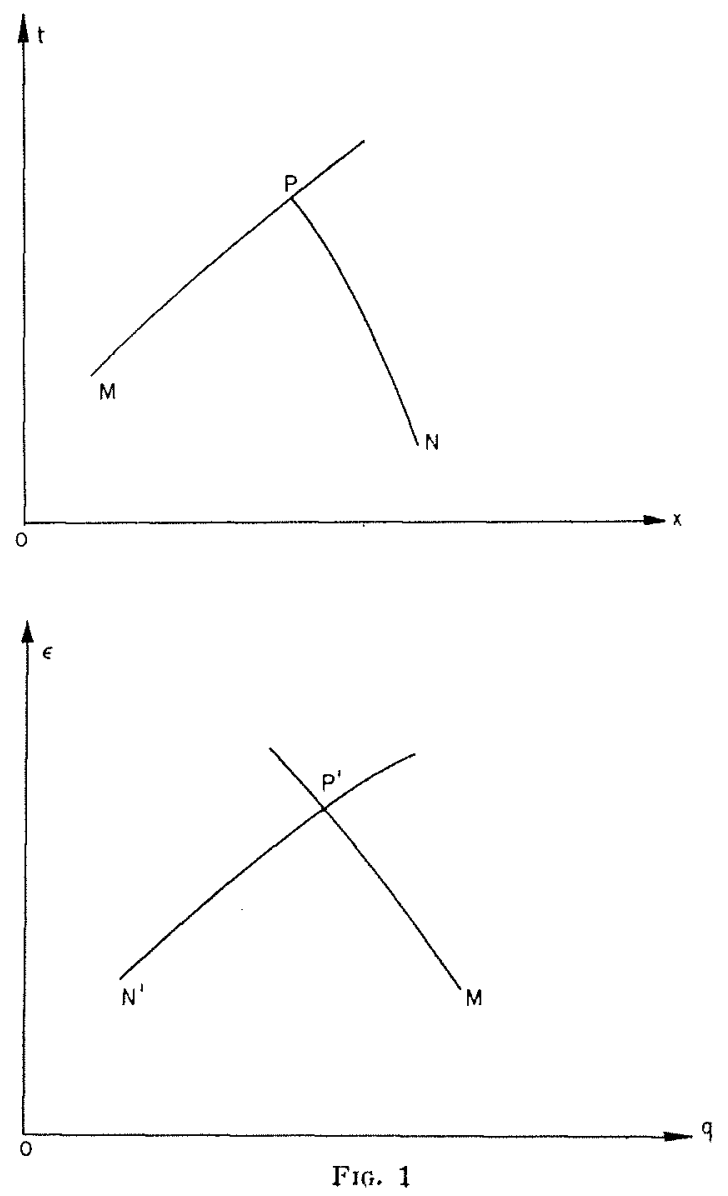
En posant $\varepsilon= \pm 1$, nous pouvons écrire, en négligeant le terme complémentaire dû à la pente et aux pertes de charge :

$$
\begin{aligned}
& \frac{d x}{d t}=\frac{x_{\mathrm{P}}-x_{\mathrm{M}}}{\mathrm{T}_{\mathrm{P}}-\mathrm{T}_{\mathrm{M}}}=(v+s c)_{\mathrm{MI}^{\mathrm{P}}} \\
& \frac{d q}{d \Sigma}=\frac{q_{\mathrm{P}^{\prime}}-q_{\mathrm{M}^{\prime}}}{\Sigma_{\mathrm{P}^{\prime}}-\Sigma_{\mathrm{X \Gamma}}}=\left(v-\varepsilon^{\prime} c\right)_{\mathrm{Mr}^{\prime} \mathrm{P}^{\prime \prime}}
\end{aligned}
$$

ct de même :

$$
\begin{aligned}
& \frac{d x}{d t}=\frac{x_{\mathrm{P}}-x_{\mathrm{N}}}{t_{\mathrm{P}^{\prime}}-t_{\mathrm{N}}}=(v-\varepsilon c)_{\mathrm{N}{ }^{\prime}} \\
& \frac{d q}{d \Sigma}=\frac{q_{\mathrm{P}^{\prime}}-q_{\mathrm{N}^{\prime}}}{\Sigma_{\mathrm{P}^{\prime}}-\Sigma_{\mathrm{N}^{\prime}}}=(v+\varepsilon c)_{\mathrm{N}^{\prime} \mathrm{P}^{\prime \prime}}
\end{aligned}
$$

Ces relations permettent de déterminer les valeurs de $t, x, q, \Sigma$ pour le point de fonctionnement $\left(\mathrm{P}, \mathrm{P}^{\prime}\right.$ à partir des valeurs des mêmes variables aux points de fonctionnement $\left(M, M^{\prime}\right)$ et $\left(\mathrm{N}, \mathrm{N}^{\prime}\right)$, ce que nous traduisons symboliquement par :

$$
\left(\mathrm{M}, \mathrm{M}^{\prime}\right)+\left(\mathrm{N}, \mathrm{N}^{\prime}\right) \rightarrow\left(\mathrm{P}, \mathrm{P}^{\prime}\right)
$$

Nous allons donner à cette relation symbolique une forme de récurrence qui sera la base de l'organisation du programme de calcul numérique.

Pour la mise en évidence de cette relation de récurrence, considérons d'abord un cas très simple, mais cependant valable dans de nombreuses applications; on suppose que :

1. Initialement, les sections afférentes aux points de fonctionnement sont équidistantes d'un intervalle $2 \Delta x$;

2. Les valeurs absolues $\left(x_{\mathrm{P}}-\cdots x_{\mathrm{M}}\right)$ ou $\left(x_{\mathrm{P}}-x_{\mathrm{N}}\right)$ sont constantes et égales à $\Delta x$;

3. Les intervalles de temps :

$$
\left(t_{\mathrm{p}}-t_{\mathrm{M}}\right) \text { ou }\left(t_{\mathrm{p}}-t_{\mathrm{N}}\right)
$$

sont aussi constants et égaux.

Ces hypothèses sont celles faites par Bergeron

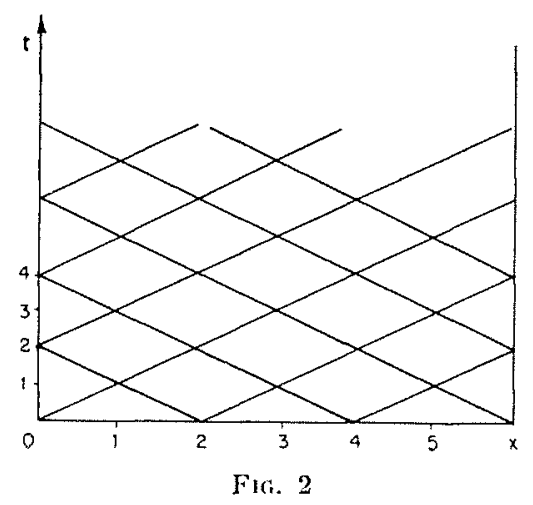

dans ses constructions graphiques relatives aux coups de bélier ou aux intumescences (3).

Dans le plan $(x, t)$, on obtient alors la représentation très simple montrée par la figure 2.

$\mathrm{M}$ est repéré par les indices $k-1, t-1$, et $\mathrm{N}$ par les indices $k+1, t-1$.

Avec les hypothèses considérées, il ne reste plus comme véritables inconnues au point $\left(P, P^{\prime}\right)$ que les valeurs de $q$ et de $\Sigma$ et le point de fonctionnement pourra être repéré par $\left(\mathrm{P}^{\prime}\right)_{k t}$ ou plus simplement, pour uniformiser les dénominations, $\operatorname{par}^{*}\left(A_{k t}\right)$.

La relation symbolique :

$$
\left(\mathrm{M}, \mathrm{M}^{\prime}\right)+\left(\mathrm{N}, \mathrm{N}^{\prime}\right) \rightarrow\left(\mathrm{P}, \mathrm{P}^{\prime}\right)
$$

devient alors :

$$
\left(\mathrm{A}_{k-1, t-1}\right)+\left(\mathrm{A}_{k+1, t-1}\right) \rightarrow\left(\mathrm{A}_{k, t}\right)
$$

relation de récurrence évidente sur le schéma simplifié de la figure, mais sur laquelle nous insistons parce qu'elle est à la base de l'organigramme de calcul.

En effet, partant des données initiales $t=0$ où tous les points $\left(A_{k, 0}\right)$ sont connus, nous calculerons tous les points de fonctionnement $\left(A_{k .1}\right)$ pour le temps 1 (ce qui correspond graphiquement à la ligne $t=1$ ). Puis, ligne par ligne, nous pourrons calculer les points pour des temps consécutifs.

Dans le cas général de deux plans de coordonnées, la relation symbolique :

$$
\left(\mathrm{A}_{k-1, t-1}\right)+\left(\mathrm{A}_{k+1, t-1}\right) \rightarrow\left(\mathrm{A}_{k, t}\right)
$$

est encore valable. Mais les indices $k$ et $t$ n'ont plus la même signification.

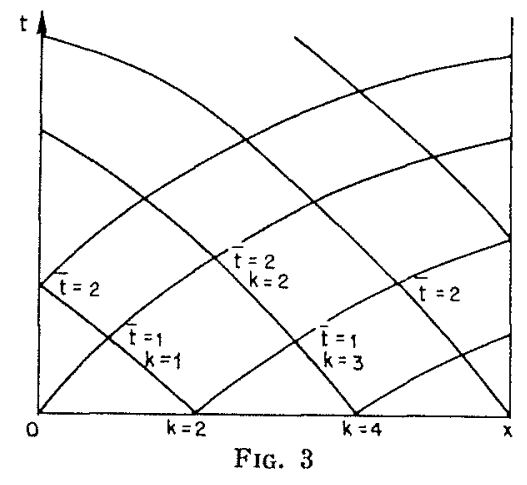

(3) Cela revient à négliger dans le seul plan $(x, t)$ la vitesse du courant devant la célérité et à prendre pour celle-ci une valeur moyenne constante. La fixité de la position des sections où s'effectue le calcul nécessite d'ailleurs une condition légèrement moins restrictive; il suffit de donner à la célérité en tous les points, la valeur moyenne $c_{\text {moy }}$ le long du canal à chaque instant. Bien entendu, on néglige encore la vitesse $v$ devant la célérité. La représentation dans le plan $(x, t)$ n'est plus formée de losanges. La valeur de $c_{\text {moy }}$ sur une ligne est donnée par la relation $c_{\text {nny }}=\sqrt{g} \overline{h_{\text {moy }}}$ 
Dans le cas précédent, ils permettaient la détermination de $x$ et de $t$; maintenant, ce ne sont plus que des numéros de référence.

La figure 3 aide à comprendre le processus.

Initialement, on connaît les points de fonctiomnement pour certaines valeurs de $x$ (qui n'ont pas besoin d'être équidistantes). L'on donne à ces points de fonctionnement un numéro d'ordre $k$ suivant les $x$ croissants, par exemple. Le temps nul est repéré par $t=0$.

A partir de deux points de fonctionnement $\left(A_{k-1,0}\right)$ et $\left(A_{k+1,0}\right)$, nous pouvons calculer le point de fonctionnement repéré par $k$. L'abscisse $x_{k}$ sera comprise entre $x_{k-1}$ et $x_{k+1}$. Le temps sera calculé par les formules aux différences finies; il sera généralement différent suivant les valeurs de $k$, mais nous pouvons le repérer quel que soit $k$ par le temps de référence $t=1$ qui exprime que ces points $\left(\mathrm{A}_{k, 1}\right)$ sont calculés, à partir des points comnus au temps $t=0$.
Ainsi, nous calculons tous les points de fonctionnement correspondant à $t=1$.

De même, nous pourrons calculer les points de fonctionnement $\left(\mathrm{A}_{k, 2}\right)$ de la ligne $t=2$ à partir des points $\left(A_{k, 1}\right)$ précédemment calculés.

Ainsi, avec la définition des numéros de référence, $k$ pour la position et $t$ pour le temps, la relation symbolique :

$$
\left(\mathrm{A}_{k-1, t-1}\right)+\left(\mathrm{A}_{k+1, t-1}\right) \rightarrow\left(\mathrm{A}_{k, t}\right)
$$

est toujours valable.

Par convention, nous désignerons dorénavant par :

$\left(A_{k, t}\right)$ le symbole du point de fonctionnement $(x, t, q, \Sigma)$;

$A_{k t}$ l'image du point de fonctionnement dans le plan $(x, t)$;

$\mathrm{A}^{\prime}{ }_{t t}$ l'image du point de fonctionnement dans le plan $(q, \Sigma)$.

\section{III. - PRINCIPE D'ORGANISATION DU CALCUL}

\section{Processus de calcul :}

D’après la relation symbolique :

$$
\left(\mathbf{A}_{k-1, t-1}\right)+\left(\mathbf{A}_{k+1, t-1}\right) \rightarrow\left(\mathbf{A}_{k, t}\right)
$$

nous avons vu que l'on pouvait effectuer le calcul ligne par ligne. Le programme de calcul sera la traduction de ce balayage de chaque ligne $t$ du point de référence $k=0$ au point de référence $k=r_{0}\left(r_{0}\right.$ étant la valeur supérieure de $k$ ).

Nous ne nous occuperons pas encore des problèmes posés par le calcul des points $\left(A_{0, t}\right)$ et $\left(A_{r_{u}, t}\right)$ situés sur les frontières, ni de la question du calcul inutile de certains points non atteints par la perturbation.

Le balayage intégral pour $k$ variant de 1 en 1 est représenté par la figure 4.

Mais nous avons préféré utiliser, comme il est traditionnel dans les épures de Bergeron, la variation de $k$ de 2 en 2 (fig. 5).

Nous reviendrons plus loin sur l'opportunité de ce changement. Le calcul de $\mathrm{A}_{k, t}$ exige la consultation des valeurs des points de fonctionnement au temps $(\bar{t}-1)$. On doit donc conserver en mémoire les résultats du calcul des points d'une ligne pour le calcul des points de la ligne suivante.

Lorsque la machine aura exécuté le calcul du dernier point de la ligne, un test devra déclencher la modification de $\bar{t}$ et le passage à la ligne suivante $\bar{t}+1$.
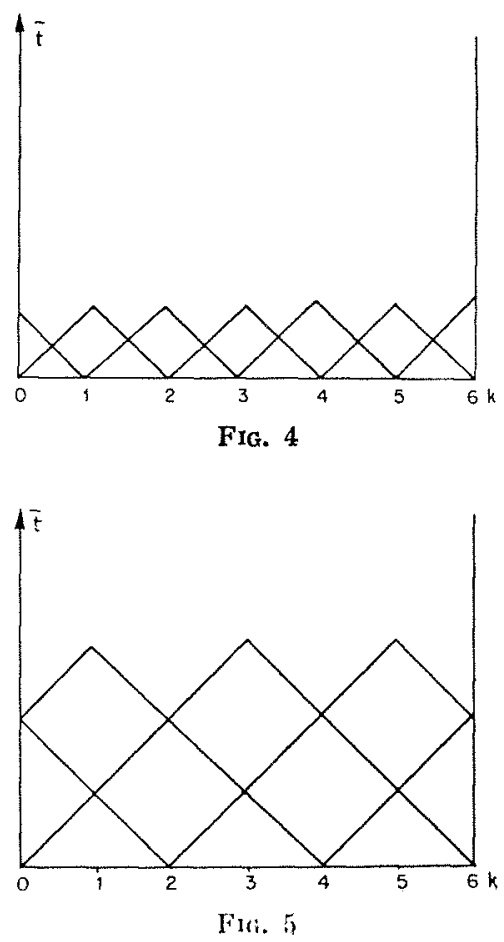


\section{Organisation du programme :}

Nous devons faire la distinction entre le calcul numérique proprement dit, exécuté conformément aux équations aux différences finies et l'organisation logique qui permet ce calcul.

Cette organisation logique remplace toutes les opéralions que fait le dessinateur quand il trace une épure de Bergeron, par exemple, choix des points de départ $A_{k-1, t-1}$ et $A_{k+1, t-1}$ pour déterminer $\left(\mathrm{A}_{k, t}\right)$, utilisation des lois particulières sur les frontières, interrogation sur le sens des variations de débits, etc.

La machine est aveugle mais douée de mémoire ef de logique acquise par l'intermédiaire du programme. Moyennant des tests ou questions, elle saura faire un choix et ainsi suivre un déroulement de calcul même très complexe.

Les tests, les modifications de programme, les transports de résultats, les ordres de perforation ou de lecture constituent l'infrastructure du programme, un squelette dans lequel rient se loger le calcul. Et remarquons que cette armature reste valable si l'on modifie partiellement les équations de base.

Pour employer une image taylorienne, l'organisation est la fonction administrative dont le calcul est la fonction opérationnelle. Nous verrons en détail la construction de cette organisation logique dans les cas particuliers étudiés plus loin.

\section{Utilisation d'ensembles de calcul :}

En général. nous rencontrerons deux sortes de points de fonctionnement :

- les points à l'intérieur du domaine $(x, t)$ définis par les limites;

- les poinls sur les frontières $k=0$ et $k=r_{0}$.

Cela nous a conduit à constituer trois enscmbles de calcul :

- Calcul général suivant la relation :

$$
\left(\mathbf{A}_{k-1, t-1}\right)+\left(\mathbf{A}_{k+1, t-1}\right) \rightarrow\left(\mathbf{A}_{k, t}\right)
$$

- Calcul particulier en $k=0$. Le calcul se fera en tenant compte des conditions particulières $\mathrm{F}_{0}(t, q, \Sigma) \stackrel{0}{=}$ existant en $k=0$ et de l'influence de $\left(A_{1, t-1}\right)$.

- Calcul particulier en $k=r_{0}$. Comme ci-dessus, le calcul se fera à partir de $\left(A_{r_{0}-1, t-1}\right)$ et $\mathrm{F}_{r_{\mathrm{o}}}(t, q, \Sigma)=0$.

Ces trois ensembles ne seront pas entièrement cloisonnés et des éléments de calcul valables pour les trois cas seront communs. Ils seront établis en sous-programme. Ainsi, le calcul lui-mène apparaîtra comme un ensemble à tiroirs.

Par exemple, le calcul de la largeur au miroir $B$ en fonction de la section mouillée $\Sigma$, la détermination du coefficient de perte de charge faisant intervenir le calcul du rayon hydraulique, le cheminement suivant des caractéristiques courbes seront communs aux trois calculs de $\left(A_{k, t}\right)$, $\left(A_{0, t}\right)$ et $\left(A_{r_{0}, t}\right)$

\section{IV. - ORGANIGRAMME DU CALCUL}

La figure 6 présente un schéma simplifié de l'organisation du calcul. La partie opérationnelle, résolution numérique des systèmes d'équations aux différcnces finies, est présentée globalenent sous la rubrique « calcul général », « calcul $\left(A_{0, t}\right) »$, «calcul $\left(A_{r_{0}, t}\right) »$, ceci à la fois pour alléger l'organigramme et pour bien mettre en évidence l'interchangeabilité de la formule de calcul.

Dans le cas général du calcul, pour les $r_{0}$ tronçons, on effectuera plusieurs boucles pour des valeurs de $k$ en progression arithmétique de raison 2. Les tests $k=r_{0}-1$ ou $k=r_{0}$ décident de la fin des boucles. Notons encore deux détails :

1. Lorsque le phénomène transitoire s'amortit par suite des frottements, les variations sont moins aiguës et l'on peut se contenter d'une moindre précision. Pour diminuer le temps de calcul, on peut faire un balayage avec $\Delta k>2$. Cette nouvelle valeur $p=\Delta k$ peut être assimilée à un pas et l'on peut la faire croître plusieurs fois dans la suite du calcul.

2. Dans le cas de manœuvre linéaire, pour se rapprocher le plus possible de la linéarité (avoir en $A_{0}, t$ des variations $\Delta q$ assez petites), on peut ne considérer qu'une fraction du canal, découpée en $r_{0}$ tronçons.

Lorsque la perturbation aura franchi cette portion de canal, on considérera une longueur plus 


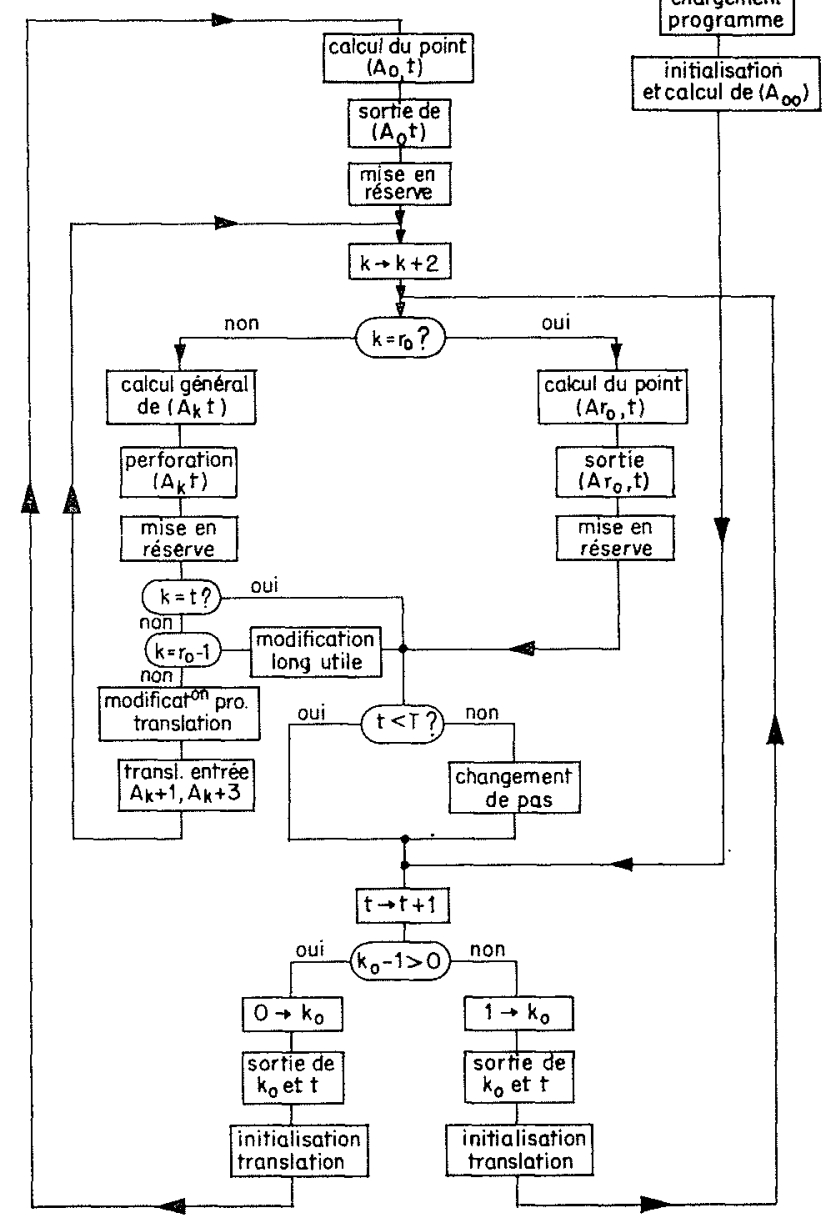

FIG. 6

grande, découpée en un même nombre de troncons. Cette opération est réalisée par l'ensemble " modification de longueur utile».
Ce schćma n'est encore qu'une ébauche et l'on peut multiplier les opérations logirues pour rendre le programme plus souple et plus nuancé.

\section{V. - ETUDE PRATIQUE DE L'INTÉGRATION NUMÉRIQUE}

Nous obtenons le point de fonctionnement $\left(A_{k}, A_{k}^{\prime}\right)$ par intersection des courbes intégrales passant par les points $\mathrm{A}, \mathrm{A}_{k-1, t-1}^{\prime}$ et $\left(\mathrm{A}, \mathrm{A}_{k+1, t-1}^{\prime}\right)$ et vérifiant les relations différentielles :

$$
\begin{gathered}
d x / d t=v \pm c: \\
(v-c) d \Sigma-d q+g \mathrm{~J} \Sigma d t=0
\end{gathered}
$$

Le seul. moyen d'intégration le plus généralement possible est l'intégration numérique. Reste alors le choix de la méthode d'intégration qui peut dépendre du découpage adopté et des valenrs des paramètres caractéristiques du cas traité (débit, pente du canal).

L'on peut décomposer la résolution du système intégral en deux parties :

- intégration des équations différentielles;

- résolution du système obtenu près de la solution en utilisant lęs différences finies. 
Soit $d \mathrm{~F}_{i}=0$ l'une des quatre équations différentielles du système $\mathrm{J}$ à intégrer.

$$
\int_{A_{k-1}}^{A_{k}} d \mathbf{F}_{i}=\int_{A_{k-1}}^{A_{k-1}^{0}} d F_{i}+\int_{\Lambda_{k-1}^{0}}^{A_{k}} d F_{i}=F_{i}\left(A_{k-1}^{0}\right)-F_{i}\left(A_{k-1}\right)+\int_{A_{k-1}^{0}}^{A_{k}} d F_{i}
$$

Si $\mathrm{A}^{(0)}{ }_{k-1}$ est suffisamment près de la solution $A_{k, t}$, on peut écrire :

$$
\int_{A^{0}{ }_{k-1}}^{A_{i}} d \mathrm{~F}_{i}=\left(\frac{d \mathrm{~F}_{i}}{d \mathrm{~A}}\right)_{\mathrm{A}^{0} k-1} d \mathrm{~A}
$$

$d$ A est caractérisé par $d x, d t$, $d a$ et $d \Sigma$ ou, si l'on passe aux différences finies, par:

$$
\begin{aligned}
& \Delta x=x_{A_{k t}}-x_{\mathrm{A}^{0} k_{t \pm 1}} \\
& \Delta t=t_{A_{k t}}-t_{A_{k \pm 1}^{0}} \\
& \Delta q=q_{A_{k t}}-q_{\Delta^{0}{ }_{k \neq 1}} \\
& \Delta \Sigma=\Sigma_{A_{k t}}-\Sigma_{\Lambda^{o} k_{t \pm 1}}
\end{aligned}
$$

Si $\mathrm{A}^{0}{ }_{k-1}$ et $\mathrm{A}^{0}{ }_{k+1}$ sont des valeurs approchées du point de fonctionnement $\mathrm{A}_{k i l}(x, t, q$, s), celuici sera solution du système :

$$
\begin{aligned}
& x-x_{A_{k-1}}+\left(t-t_{A^{0} k_{k-1}}\right)(v+c)_{A_{k i-i}^{0}}+\int_{A_{k-1}}^{A_{k-1}^{0}}(v+c) d t=0 \\
& x-x_{A_{k+1}}+\left(t-t_{A^{0} k_{k+1}}\right)(\nu-c)_{A_{k+1}^{0}}+\int_{A_{k+1}}^{A^{0} k_{k+1}}(\nu-c) d t=0
\end{aligned}
$$

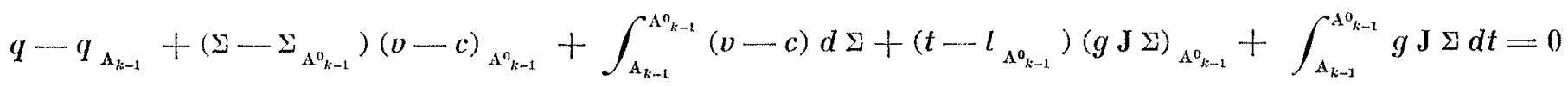

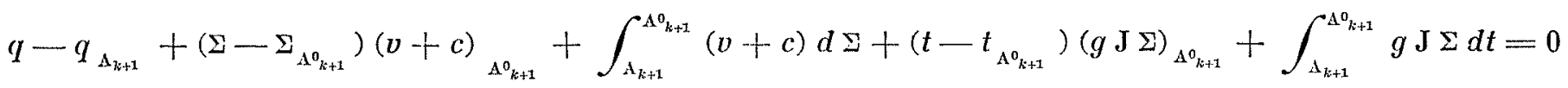

La valeur ainsi trouvée pourra servir de point de départ à une itération.

Pour éviter la résolution d'un système de quatre équations aux inconnues $x, t, q, \Sigma$, on peut décomposer l'itération en deux opérations portant successivement sur le couple $(x, t)$, puis sur le couple $(q, \Sigma)$.

Considérons le système différentiel :

$$
d \Sigma-\frac{d q}{v \pm c}+\frac{d t}{v \pm c}(g \mathrm{~J} \Sigma)=0
$$

Pour alléger l'écriture, posons :

$$
\mathrm{D}=g \mathrm{~J} \Sigma=g_{i} \Sigma-\frac{k q^{2}}{\Sigma}+\frac{1}{\mathrm{~B}} \frac{\partial \Sigma}{\partial \alpha} \frac{\partial \alpha}{\partial x}
$$

On divise l'intégration des équations ci-dessus en deux parties :

$$
\int\left(d \Sigma-\frac{d q}{v \pm c}\right)+\int_{A_{k-t}}^{A_{k t}} \frac{\mathrm{D}}{v \pm c} d t=0
$$

La seconde intégrale sera calculée par une moyenne pondérée :

$$
\int \frac{\mathrm{D} d t}{v \pm c}=\int \frac{\mathrm{D} d x}{v^{2}-c^{2}}=\left[\frac{\mathrm{D}}{v^{2}-\cdots c^{2}}\right]_{\text {woy. }}^{\Delta x}
$$

On peut ainsi choisir l'expression simple :

$$
\int \frac{\mathrm{D}}{v^{2}-c^{2}} d x \cong \frac{\Delta x}{2}\left[\left(\frac{\mathrm{D}}{v^{2}-c^{2}}\right)_{A_{k-1, t} t-1}+\left(\frac{\mathrm{D}}{v^{2}-c^{2}}\right)_{\mathrm{A}_{k, t}}\right]
$$


L'intégrale $\int d \Sigma-[d q /(v+c)]$ peut être calculée à l'aide d'une formule classique d'intégration pas à pas. On peut, par exemple, employer la formule de Runge Kutta utilisée dans la déterminalion des courbes de remous. Mais la précision de cette méthode n'est pas nécessaire, car l'on ne doit pas oublier que les hypothèses simplificatrices de base introduisent des causes d'imprécision, et il est inutile de rechercher une exactitude mathématique qui n'aurait aucun rapport avec les possibilités de vérification expérimentale. L'on peut se contenter de la formule de la tangente ou au plus de la méthode d'Euler.

Le cheminement, le long des courbes, reste encore valable dans le cas de l'hypothèse d'une laible incidence du terme complémentaire $\Delta t[\mathrm{D} /(v+\varepsilon c)]$. Car, en toute rigueur, l'on ne pent séparer l'intégration de l'équation (5) en deux parties. Il y a interférence entre les valeurs de $\mathrm{D}$ et de $\Sigma$ tout au long des courbes intégrales. Néanmoins, dans la plupart des cas traités, l'hypothèse simplificatrice se trouve bien vérifiée.

\section{VI. - MÉTHODE NUMERIQUE SIMPLIFIEE N'UTILISANT QU'UN PLAN DE COORDONNEES}

Dans l'étude sommaire de certains problèmes ou dans l'examen systématique d'avant-projet, il peut être intéressant de connaître rapidement l'évolution des phénomènes au cours du temps dans une section ou le long de l'ouvrage à une late déterminće.

\section{Principe et kypothèses}

La méthode numérique d'étude d'intumescences, précédemment exposée, nécessite pour l'exploitation des résultats de porter les valeurs des points de fonctionnement dans le plan $(x, t)$ et de procéder à des interpolations.

Ce but est atteint dans la méthode n'utilisant que le plan de coordonnées $(\Sigma, q)$. Les hypothèses simplificatrices ont déjà été signalées dans la mise en évidence de la formule de récurrence de base.

1. La position, le long du canal des profils où s'effectue le calcul, est fixe.

2. Le temps est déterminé approximativement à partir de la valeur moyenne de la célérité, à chaque instant, tout au long du canal.

$$
\Delta t\left(x_{i}\right)=\frac{\Delta x}{c_{m(t)}}
$$

Ces deux hypothèses montrent que, dans le plan $(x, t)$, on néglige l'influence de la vitesse $v$ du courant devant la célérité des ondes, que l'on considère indépendante de l'abscisse. Mais, notons que ces restrictions sont faites seulement pour les opérations portant sur le plan $(x, t)$ et non le plan $(q, \Sigma)$.
Cetle méthode numérique est, avec l'utilisation des variables $(q, \Sigma)$, l'analogue de la méthode graphicque des caractéristiques courbes mise au point par l'un de nous.

\section{Critiques et hypothèses}

Les hypothèses admises faussent la position dans le plan des points de fonctionnement. Cette erreur a évidemment une incidence sur la détermination des valeurs de $\Sigma$ et de $q$ (à cause des pertes de charge, de la non-uniformité possible du régime initial et des singularités éventuelles du profil du canal) par l'intermédiaire du terme $\Delta t[\mathrm{D} /(v \pm c)]$.

Nous étudierons plus loin l'importance de l'influence de ces hypothèses et l'évolution de l'erreur commise sur la détermination des points $A_{(x, t)}$. Nous indiquerons aussi une amélioration possible dans la détermination du temps.

Cependant, nous pouvons déjà affirmer que, dans la plupart des cas rencontrés dans la pratique industrielle, cet écart d'avec une détermination plus rigoureuse de la position de $\mathbf{A}_{(x, t)}$ reste dans des limites acceptables. La meilleure preuve est la très bonne concordance des résultats d'essais numériques avec les mesures expérimentales.

\section{Réalisation pratique}

La seule partie du programme à modifier est le sous-programme de calcul de $x$ et $t$ dans l'ensemble calcul général.

$x$ étant déterminé par $k$, il suffit de calculer une valeur approximative de $t$. 
On suppose que les dates de départ des ondes de $A_{k-1, t-1}$ et $A_{k+1, t-1}$ sont identiques. Dans ces conditions, l'intervalle de temps sera environ :

$$
\Delta t=\frac{\Delta x}{2}\left(\frac{1}{v+c}-\frac{1}{v-c}\right)
$$

et l'on admettra pour valeur de $t$ au point $A_{k, t}$ :

$$
t=\frac{t_{A_{k-1}}+t_{A_{k+1}}}{2}+\frac{\Delta x}{2}\left(\frac{1}{v+c}-\frac{1}{v-c}\right)
$$

Cette valeur présente deux défauts :

- elle n'est pas rigoureusement exacte;

- elle n'est pas cohérente avec les hypothèses simplificatrices de base.

En effet, celles-ci postulent que le temps est le même pour tous les points $A_{k}$ d'une même ligne $\tilde{t}$ et égale à $t_{\text {moy }}$.

Mais la formule proposée présente cependant un intérêt. Etant plus proche de la réalité que la valeur $t_{\text {moy, }}$ elle permet d'apprécier l'ordre de grandeur de l'approximation consentie pour l'emploi de la méthode simplifiée.

De plus, il sera mis en évidence plus loin que cette détermination du temps ne détruit pas la simplification introduite par les hypothèses rappelées précédemment.

\section{Emploi de caractéristiques rectilignes dans le plan}

Lorsqu'on veut ajouter à la simplicité du dépouillement, la réduction du temps de calcul, on peut supprimer le cheminement le long des caractéristiques courbes.

Cette économie peut être obtenue de deux manières :

- en shuntant dans le programme le sousprogramme d'intégration pas à pas sur les caractéristiques du plan $(q, \Sigma)$;

- en introduisant une valeur du pas $\Delta q$ supérieure à l'intervalle de variation du débit. Ainsi le test $q_{A_{k-1}}-q_{\Lambda^{0} k}<\Delta q$ dirigera toujours vers la dernière opération qui est la détermination linéaire du point de fonctionnement.

D'après la confrontation des résultats obtenus avec les essais sur modèle réduit, l'emploi de caractéristiques rectilignes donne des résultats peu précis, mais, dans le sens de la sécurité, en ce qui concerne les ondes positives. Cette méthode rapide convient donc pour une ctude de lordre de grandeur des surélévations dues au passage d'une intumescence.

\section{Comparaison de la méthode simplifiée et de la méthode complète}

La méthode fondée sur l'emploi de profils de calcul fixes est moins rigoureuse que la méthode générale utilisant quatre coordonnées $x, t, q, \Sigma$, pour définir un point de fonctionnement.

Les résultats obtenus par la méthode simplifiée sont moins précis, surtout en ce qui concerne le temps, que ceux fournis par la méthode complexe. Mais l'exactitude permise est généralement suffisante dans la pratique, surtout si l'on remarque que les hypothèses fondamentales et le fait de négliger les oscillations secondaires introduisent des causes d'approximations importantes dès l'établissement des équations de base de la théorie générale.

Les avantages principaux présentés par l'emploi de la méthode simplifiée sont les suivants :

- dépouillement rapide et aisé des essais numériques : la lecture des résultats permet la connaissance immédiate de l'évolution de l'écoulement en un profil en fonction dı temps;

- adaptation au cas de cours d'eau naturel en introduisant dans le groupe de mémoiresréserve d'un profil des paramètres caractéristiques d'un tronçon, largeur au radier, fruil des berges, coefficient de Strickler;

- localisation des singularités. Pour faciliter le calcul, il suffit de conserver dans le groupe des cellules-réserves, les grandeurs caractéristiques à gauche et à droite (ou amont el aval) du profil singulier.

Il y a donc intérêl, avant toute étude numérique, de juger si la distorsion due à la méthode simplifice est acceptable. 


\section{VII. - CONDITIONS AUX LIMITES}

\section{Détermination du point de fonctionnement en front d'onde}

La méthode d'intégration qui précède s'applirue au calcul du point de fonctionnement en corps d'onde ou éventuellement en front d'onde lorsque celui-ci est peu incliné.

Des modifications sont à introduire lorsque la lole de l'intumescence est abrupte.

Le programme général de calcul est encore valable, mais il sera modifié en deux points : valeur de la célérité et évaluation du terme D.

Les équations définissant le mouvement du iront d'onde sont apparemment différentes des équations régissant le mouvement en corps d'intumescence résumées dans le système:

$$
\left\{\begin{array}{l}
\frac{d x}{v \pm c}=d t \\
(v \pm c) d \Sigma-d \eta+\mathrm{D} d t=0
\end{array}\right.
$$

Dans le plan $(x, t)$, la première équation est encore valable pour la propagation du front d'onde sauf à modifier la valeur de $c$.

Il faut remplacer la vitesse $c=\sqrt{g(\Sigma / \mathrm{B})}$ d'une onde superficielle par la célérité $c_{f}$.

L'équation de continuité $\left(v_{0} \doteq c_{f}\right) \Delta \Sigma=\Delta Q$ est la traduction en différences finies de la seconde relation différentielle où l'on néglige le terme D $d t$.

Cette analogie formelle suggère la possibilité d'utiliser le programme existant pour le calcul des conditions de fonctionnement en front d'onde.

\section{Calcul des conditions de fonctionnement initiales}

Le régime initial est caractérisé par une courbe de remous. La détermination de la ligne d'eau initiale s'cffectuera par intégration pas à pas de l'équation différentielle caractérisant le régime graduellement varié.

Cette équation, déjà rencontrée traitant des courbes de remous est :

$$
\frac{d \Sigma}{d x}=\frac{=g(i-j+l) \Sigma}{v^{2}-c^{2}}
$$

ou, avec les notations déjà employées:

$$
\frac{d \Sigma}{d x}=\frac{\mathrm{D}}{m n}
$$

La programmation du calcul de $d \Sigma / d x$ sera facilitée par l'emploi des sous-programmes de calcul de $D, m$ et $n$ existant déjà dans le programme général.

$x$ déterminée par $h \Delta x$ varie de 0 à $r_{0} \Delta x=\mathrm{L}$.

Le calcul des valeurs des fonctions cherchées se fait dans les cellules-travail et les résultats sont envoyés dans les cellules-réserves correspoudant à la valeur de $k$.

\section{Calcul des points de fonctionnement aux limites}

La détermination des grandeurs $t, q$ et $\Sigma$ dans les sections amont et aval dépend des conditions de fonctionnement particulières en ces points.

$t$ sera détermine par intersection de la caractéristique $(x, t)$ issue du point $\mathrm{A}_{k, t-1}$ et de la loi $x=\mathrm{C}^{\mathrm{te}}$.

$\Sigma$ et $q$ seront solution du système : $\mathrm{F}(q, \Sigma, t)=0$ condition à la limite

$$
\int d \Sigma+\int \frac{d q}{v \pm c}+\int \frac{\mathrm{D} d x}{v^{2} \pm c^{2}}=0
$$

\section{Examens des conditions aux limites les plus fréquemment rencontrées}

1. Section De mandeuve $k=0$ :

Généralement, on impose une loi de débit:

a) $q=\mathrm{C}^{\text {te }}$ est la plus simple, mais aussi la plus fréquente, par exemple dans le cas du déclenchement brusque, $q=0$. C'est l'hypothèse généralement la plus défavorable au point de vue sécurité et son étude permet de prévoir les limites de surélévations.

b) $q=f(t)$. La forme de la loi la plus couramment rencontrée est la variation linéaire du débit.

Le débit sera alors caractérisé par le débit $q_{0}$ à l'instant initial $t=0_{+}$et l'accélération $\%$, tel que $q=q_{0}+\gamma t$.

c) Manouvres superposées. - Si l'on considère le cas d'une usine où l'on effectue des prises de charge entrecoupées de déclenchements hrusques, la variation du débit peut être repré- 


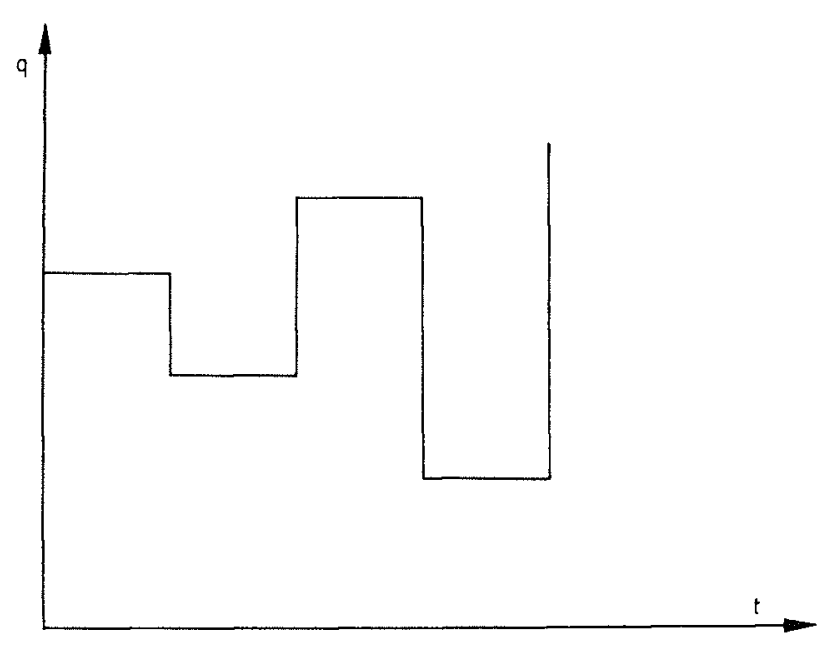

Fic. $i$

sentée par la figure 7. L'amplitude des paliers et des variations dépend du nombre de groupes en fonctionnement et en régime instable (déclenchement). La loi de débit, même complexe, peut être mise en mémoire dans l'ordinateur, mais il est plus simple de prévoir une lecture de la valeur du débit $q_{(0, t)}$ lorsque va s'effectuer le calcul du point $(t, q \Sigma)$ de la section de manœuvre.

\section{INFLUENCE D'UN DÉVERSOIR :}

La condition à la limite, ici de forme $f(q \Sigma)=0$ a pour expression :

$$
\mathrm{Q}=k \mathrm{~L} h \sqrt{2 g h}=k \mathrm{~L} \sqrt{2 g} h^{3 / 2}(\Sigma)
$$

On emploie toujours le même processus :

détermination d'une valeur approchée par intersection de la caractéristique rectiligne issue de $\mathrm{A}_{r_{0}-1, t-1}$ et de la courbe $f(Q, \Sigma)=0$;

- cheminement sur une caractéristique courbe jusqu'à proximité de la solution;

-- détermination de la solution comme intersection d'un segment rectiligne et de la courbe $f(\mathbf{Q}, \Sigma)$ à l'aide du programme déjà utilisé pour obtenir la solution approchée.

L'élément nouveau est donc la résolution du système :

$$
\begin{aligned}
\Sigma-\Sigma_{A_{r_{0}-1}^{n_{n}}} & =\frac{Q-Q_{A_{r_{v}-1}}}{m !}+\frac{\left(t-t_{A_{r_{0}-1}}\right) D_{m 0 x}}{n} \\
Q & =k L \sqrt{2 g} h^{3 / 2}(\Sigma)
\end{aligned}
$$

$\mathrm{Si}\left(\partial \Sigma^{(i)} / \partial \Sigma^{(i-1)}\right)<1$,

c'est-à-dire $[(k \mathrm{~L} \sqrt{2 g} / \mathrm{m}][(3 / 2) h][\partial h / \partial \Sigma]<1$, on peut employer une itération qui donnera à la fois $Q$ et $\Sigma$.

\section{INFLUENCE D'UNE VANNE DE FOND :}

La loi de débit de la vanne est $[q, \Sigma(t), t]$ ou $f(q, \Sigma, t)$ le temps intervenant dans la loi de levée de la vanne.

On peut encore utiliser le procédé ci-dessus en faisant intervenir dans l'itération le temps qui variera peu.

\section{Passage en charge d'une intumfscence:}

Deux cas intéressants de passage en charge d'une intumescence sont le raccordement d'un canal à écoulement libre avec une galerie et le passage en siphon entre deux canaux.

a) Jonction d'un canal et d'une galerie d'amenée en charge. - Avec les notations représentées

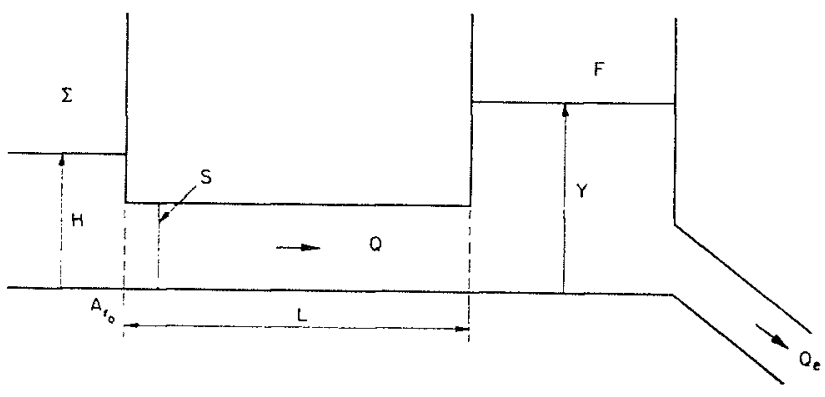

FIG. 8

sur la figure 8, le système obéit au système d'équations :

$$
\begin{gathered}
d \Sigma-\frac{d \mathrm{Q}}{d-c}+\frac{\mathrm{D} d x}{v^{2}-c^{2}}=0 \\
\frac{\mathrm{L}}{g \mathrm{~S}} \frac{d \mathrm{Q}}{d t}=\mathrm{H}-\mathrm{Y}-c \mathrm{Q}^{2} \\
\mathrm{~F} \frac{d \mathrm{Y}}{d t}=\mathrm{Q}-\mathrm{Q}_{e}
\end{gathered}
$$

Comme $\Sigma$ et $H$ sont liés de manière biunivoque, ce système définit les trois inconnues $\mathrm{Q}, \mathrm{H}$ et $\mathrm{Y}$, compte tenu des conditions initiales.

La condition à la limite en $A_{r_{0}}$, désignée par $\mathrm{F}_{r_{0}}(l, q, \Sigma)$, qui s'exprimait précédemment au moyen d'une équation algébrique, est maintenant traduite de manière plus complexe par les équations :

$$
\begin{gathered}
\frac{\mathrm{L}}{g \mathrm{~S}} \frac{d \mathrm{Q}}{d t}=\mathrm{H}-\mathrm{Y}-c \mathrm{Q}^{2} \\
\mathrm{~F} \frac{d \mathrm{Y}}{d t}=\mathrm{Q}-\mathrm{Q}
\end{gathered}
$$

Mais le principe de résolution est toujours valable. Le point de fonctionnement $\left(A_{r_{0}, t}\right)$ est déterminé à partir de $\left(\mathrm{A}_{r_{0}-1, t-1}\right)$ et $\mathrm{F}_{r_{0}}\left(t, q, q^{\prime}, \Sigma\right)$. Seule, la partie du programme afférente au 
«calcul $A_{r_{0}}$ » sera modifiée de façon à effectuer la résolution du système différentiel.

Le calcul de $\left(A_{r_{0}, t}\right)$ ne fait intervenir que des cellules - réserve dont on connait la position, le stockage des fonctions supplémentaires $\mathrm{Y}, \mathrm{Q}_{c}$ qu'il faut consulter n'introduit donc aucune difficulté.

La résolution du système différentiel sera effectuće par itération. Une première approximation localise la solution. Ensuite, après avoir effectué un cheminement sur les courbes intégrales, on peut, au voisinage de la solution, linéariser les équations et les transformer, après passage aux différences finies, en un système linéaire facile à résoudre.

b) Siphon reliant deux portions d'un même canal. - Le problème du passage en siphon d'une intumescence présente une analogie formelle arec Je problème précédent. Cependant, une distinction s'impose. Il ne s'agit plus de condition à la limite, mais d'existence d'une singularité en cours d'ouvrage.
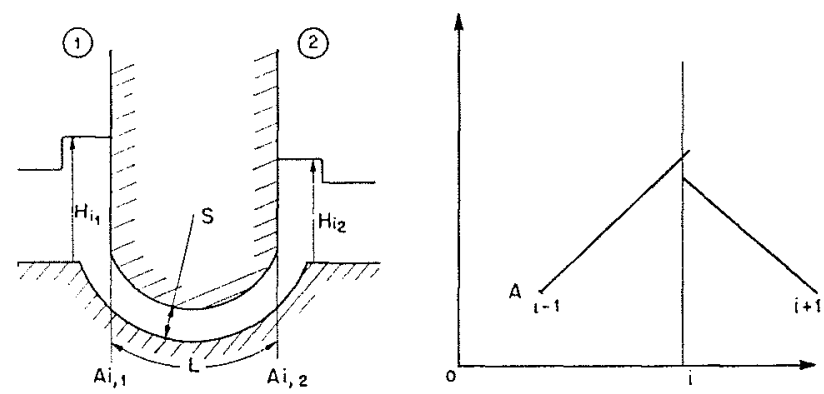

FIG. 9

Avec les notations de la figure 9, les équations régissant le fonctionnement du siphon sont les suivantes :

$$
\begin{gathered}
\frac{\mathrm{L}}{g \mathrm{~S}} \frac{d \mathrm{Q}}{d t}=\mathrm{H}_{1}-\mathrm{H}_{2}-c \mathrm{Q}^{2} \\
d \Sigma_{1}-\frac{d \mathrm{Q}}{v_{1}-c_{1}}+\frac{\mathrm{D}_{1} d x}{v_{1}^{2}--c_{1}^{2}}=0 \\
d \Sigma_{1}-\frac{d \mathrm{Q}}{v_{2}+c_{2}}+\frac{\mathrm{D}_{2} d x}{v_{2}^{2}--c_{2}^{2}}=0
\end{gathered}
$$

Notons que, dans ce cas, il apparaît deux équalions dues aux caractéristiques, ce qui va compliquer le problème, car il faut calculer en même temps les points de fonctionnement en $A_{i, 1}$ et $A_{i, 2}$ et rien ne permet d'assurer, a priori, que dans le plan $(x, t)$ les caractéristiques issues de $A_{i-1, t-1}$ et $A_{i+1, t-1}$ se rencontrent sur la droite $x=x_{A i}$.

Si on désigne par $\mathrm{F}_{s}\left(\mathrm{Q}, \mathrm{H}_{1}, \mathrm{H}_{2}, t\right)=0$ l'équa- tion propre au siphon, le calcul de $\mathbf{A}_{i_{t} t}$ s'effectue suivant le schéma :

$$
\begin{aligned}
& \left(A_{i-1, t-1}\right)+\left(A_{i+1, t-1}\right) \\
& +F_{s}\left(Q_{i}, H_{i_{1}}, H_{i, 2}\right) \rightarrow\left(A_{i_{1}, t} A_{i_{2}, t}\right)
\end{aligned}
$$

Dans le traitement numérique, le siphon peul se réduire à une singularité ponctuelle. Tout sc passe alors comme si l'on avait un profil unique où se produirait une discontinuité, avec des vaieurs différentes à droite et à gauche. Il est alors nécessaire pour ce profil de mettre en réserve deux groupes de valeurs $\mathrm{Q}, \Sigma, v, c$ correspondant aux caractéristiques $d u$ fonctionnement du point immédiatement à l'amont et immédiatement à l'aval.

On peut d'ailleurs généraliser cette facon de procéder à la plupart des singularités (pỉles de ponts, prises d'eau). La distinction entre valeurs à droite et valeurs à gauche est particulièrement utile dans le cas de modification de section.

Revenons à la détermination du point de fonctionnement $\left(\mathrm{A}_{i_{1}, t} \mathrm{~A}_{i_{\text {g. }}, t}\right)$.

Suivant la disposition dans le plan $(x, t)$ des caractéristiques issues de $A_{i-1}$ et $A_{i+1}$, il faut opérer une interpolation ou non.

Un point important dans la préparation du cal. cul est l'incidence du siphon sur le découpage du canal.

Si nous choisissons une méthode du second ordre, l'équation dynamique du siphon entre les instants $t-1$ et $t$ se traduit par :

$$
\Delta \mathrm{Q}_{s}=\frac{g S}{\mathrm{~L}} \cdot \frac{1}{2}\left(\varphi_{t-1}+\varphi_{t}\right) \Delta t
$$

en posant :

$$
\mathrm{H}_{i, 1}-\mathrm{H}_{i, 2}-c \mathrm{Q}^{2}=\varphi
$$

Une condition est à respecter : il ne faut pas que la variation $\Delta \mathrm{Q}_{s}$ de débit soit trop grande et, en particulier, supérieure à la variation totale due à la manœuvre.

Essayons de préciser cette condition : la valeur de $\Delta Q$. maximale correspond à la valeur maximale de $d \mathrm{Q} / d t$, soit :

$$
\left(\frac{a^{3} \mathrm{Q}}{d t}\right)_{\max }=\frac{g \mathrm{~S}}{\mathrm{~L}}\left(\mathrm{H}_{1}-\mathrm{H}_{2}\right)_{\max }=\frac{g \mathrm{~S}}{\mathrm{~L}} \Delta \mathrm{H}
$$

$\Delta \mathrm{H}$ est la surélévation maximale due à l'intumescence.

Or, d'après l'équation de continuité des intumescences :

$$
\Delta \mathrm{Q}_{\max } \# c \Delta \Sigma \# c B \times \Delta \mathrm{H}
$$

il vient donc :

$$
\frac{\Delta \mathrm{Q}_{\text {siphoH }}}{\Delta \mathrm{Q}_{\operatorname{maxi}}}=\frac{g(\mathrm{~S} / \mathrm{L}) \Delta \mathrm{H} \Delta t}{c \mathrm{~B} \Delta \mathrm{H}}=\frac{g \mathrm{~S} \Delta x}{\mathrm{LB}:^{2}}
$$


La condition à vérifier $\Delta Q_{\text {siphon }} / \Delta Q_{\max }<1$ se traduit donc par $(\mathrm{S} / \Sigma)(\Delta x / \mathrm{L})<1$.

En fait, dans le cas d'une détermination du second ordre :

$$
\Delta Q_{s}=\frac{g S}{L} \frac{1}{2}\left(\varphi_{0}+\varphi_{1}\right)
$$

Si $\Delta Q_{s}$ est maximum, $\varphi_{1} \# 0$; donc, il suffit que :

$$
\frac{\mathrm{S}}{\mathrm{\Sigma}} \frac{\Delta x}{\mathrm{~L}}<<2
$$

Cette inégalité permet de vérifier si la valeur du découpage choisie est correcte.

\section{Exemple numérique}

Soit un canal à profil trapézoïdal dont les caractéristiques sont :

-. longueur ............. $5 \mathrm{~km}$

- pente .............. 2,5\%

- largeur au radier.......... $5 \mathrm{~m}$

- tirant d'eau normal......... 3,60 m

- fruit des berges............ 2

- débit ............. $70 \mathrm{~m}^{3} / \mathrm{s}$

Un siphon de $340 \mathrm{~m}$ de long et de $25 \mathrm{~m}^{2}$ de section assure le passage d'un thalveg. Pour que le calcul soit valable, il faut envisager un découpage qui peut être déterminé à l'aide d'un calcul relativement simple et tenant compte de la présence d'un siphon.

Ici, il faut donc diviser le canal au minimum en huit tronçons. Si l'on désire avoir une variation plus continue du débit à la traversée du siphon, il faut, bien entendu, choisir un nombre de tronçons plus grand.

\section{Conclusions :}

Aux limites, le calcul des points de fonctionnement s'effectue toujours suivant le même schéma symbolique :

$$
\left(\mathrm{A}^{1}{ }_{r_{0}-1, t-1}\right)+\mathrm{F}(\Sigma, q, t, y, z) \rightarrow\left(\mathrm{A}^{0}{ }_{r_{0}, t}\right)
$$

$y, z$ représentant des grandeurs extérieures au canal (par exemple niveau dans une chambre d'équilibre, ouverture d'une vanne, etc.).

La loi de fonctionnement sur la limite $\mathrm{F}(\Sigma, q, t, y, z)=0$ peut se présenter sous la forme d'expressions algébriques plus ou moins compliquées (par exemple $q=\mathrm{C}^{\mathrm{te}}, q=m(t) \mathrm{H}^{3 / 2}$ ) ou sous la forme de relations différentielles uniques ou multiples.

Dans tous les cas, la méthode de résolution la plus générale et la plus aisée à mettre en œuvre est un procédé itératif dont il faut évidemment vérifier la convergence.

L'itération peut se décomposer en plusieurs phases successives. Il faut d'abord chercher une solution approchée à l'aide d'une méthode du premier ordre, par exemple.

Puis, au voisinage de la solution, on transforme les équations algébriques ou différentielles en un système d'équations linéaires obtenues en utilisant des différences finies.

\section{VIII. - EXEMPLES D'APPLICATION}

Le procédé de calcul précédemment exposé permet d'aboutir à deux méthodes numériques :

1. Lune d'elles, méthode simplifiée, correspond à l'hypothèse, citée plus haut, de la fixité de la position des sections de calcul;

2. L'autre est l'application intégrale de la théorie des caractéristiques.

Nous ne pouvons pas, dans le cadre de cette communication, exposer en détail chacune d'elles. Toutefois, il nous semble utile de fournir deux exemples d'utilisation.

\section{Etude par la méthode simplifiée d'une fer- meture brusque d'un débit à l'aval d'un canal de section trapézoïdale}

L'ouvrage présente les caractéristiques suivantes :

- longueur du canal........ $5700 \mathrm{~m}$

- section trapézoïdale : largeur au radier............. $5,50 \mathrm{~m}$

- fruit des berges........... $3 / 2$

- débit .............. $130 \mathrm{~m}^{3 / \mathrm{s}}$ 

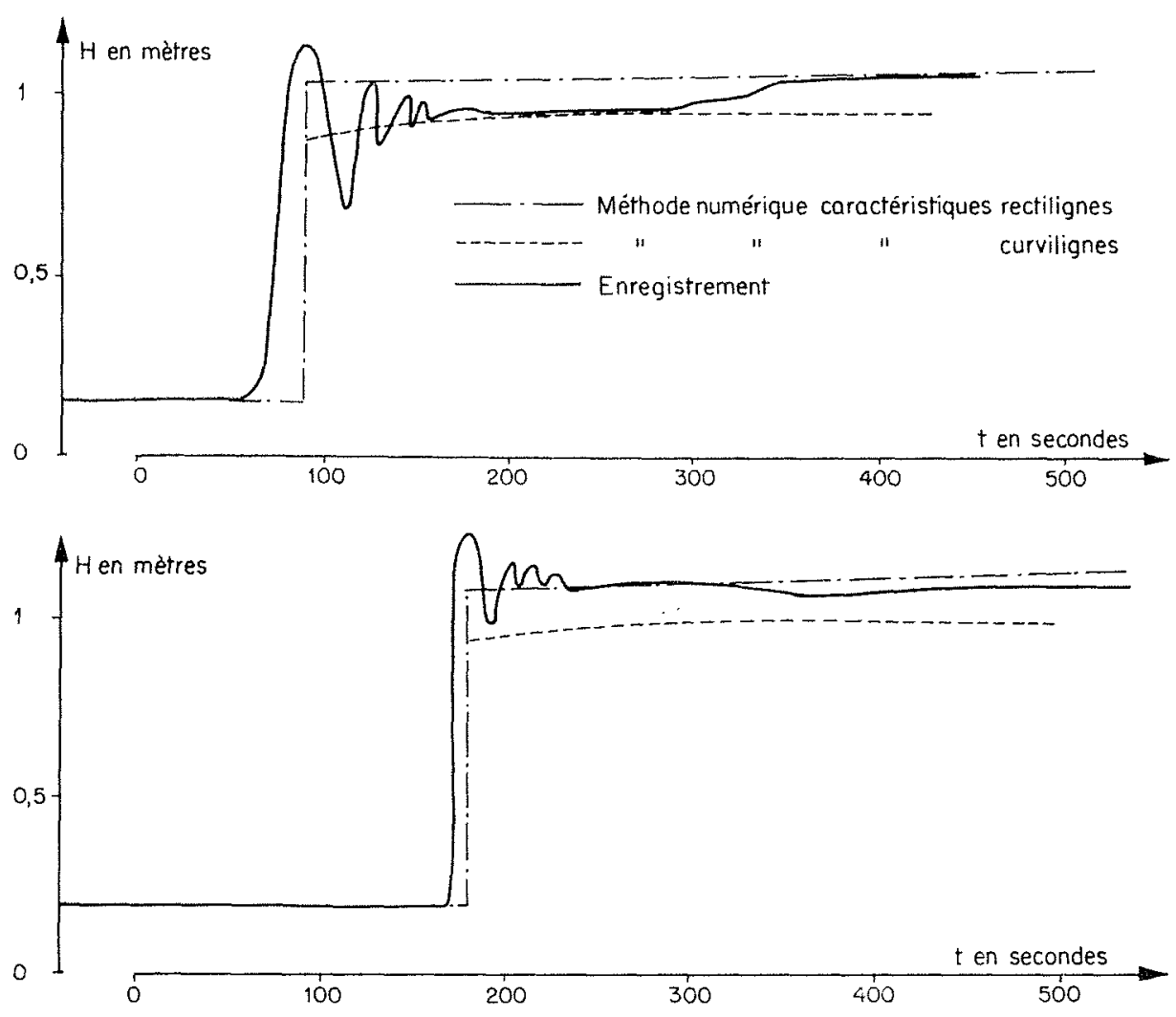

FIC. 10

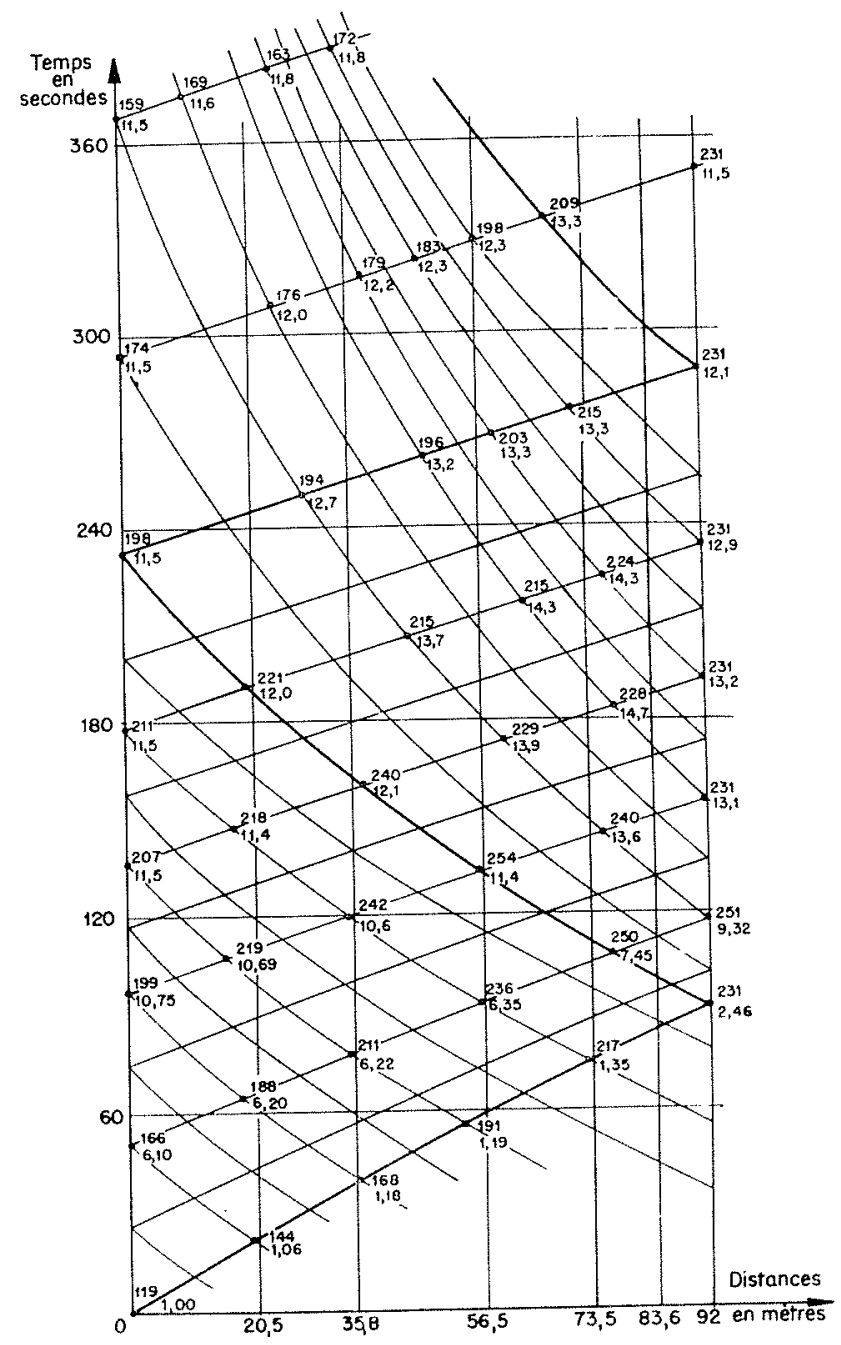

FIG. 11 
— coefficient de Strickler...... $\quad k=57$

- pente du radier.......... $i=0,15 \%$

L'état initial est caractérisé par la ligne d'eau $H(x)$ correspondant à un régime graduellement varié.

La figure 10 établit la comparaison entre les résultats obtenus soit par expérience sur modèle réduit, soit par calcul numérique.

Les courbes $H(t)$ ont été tracées pour deux sections respectivement situées à $570 \mathrm{~m}$ et $1140 \mathrm{~m}$ de l'aval.

\section{Etude par la méthode complète d'une ou-} verture lente de débit à l'amont d'une galerie de fuite de profil circulaire

Les résultats présentés correspondent à une ouverture très lente, car les manœuvres rapides provoquent, dans les cas considérés, la mise en charge de la galerie dans le tiers aval.
Les caractéristiques de la galerie sont les suivantes :

- longueur . . ............ $92 \mathrm{~m}$

- diamètre . . . . . . . . . . . 0,20

_. coefficient de Strickler.......... 100

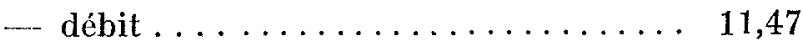

- durée d'ouverture............. 100 s

La figure 11 représente le plan $(x, t)$ où ont été reportés les résultats $\bar{x}, t, \Sigma, q$ de l'essai numérique. On notera la courbure des caractéristiques. Par interpolation, on a déduit les courbes $h(t)$ dans plusieurs sections (fig. 12) et les profils en long à divers instants (fig. 13). Ces deux cas montrent ainsi nettement l'intérêt de ces méthodes numériques : le premier, par la concordance entre résultats théoriques et expérimentaux, le second par la simplification du calcul.
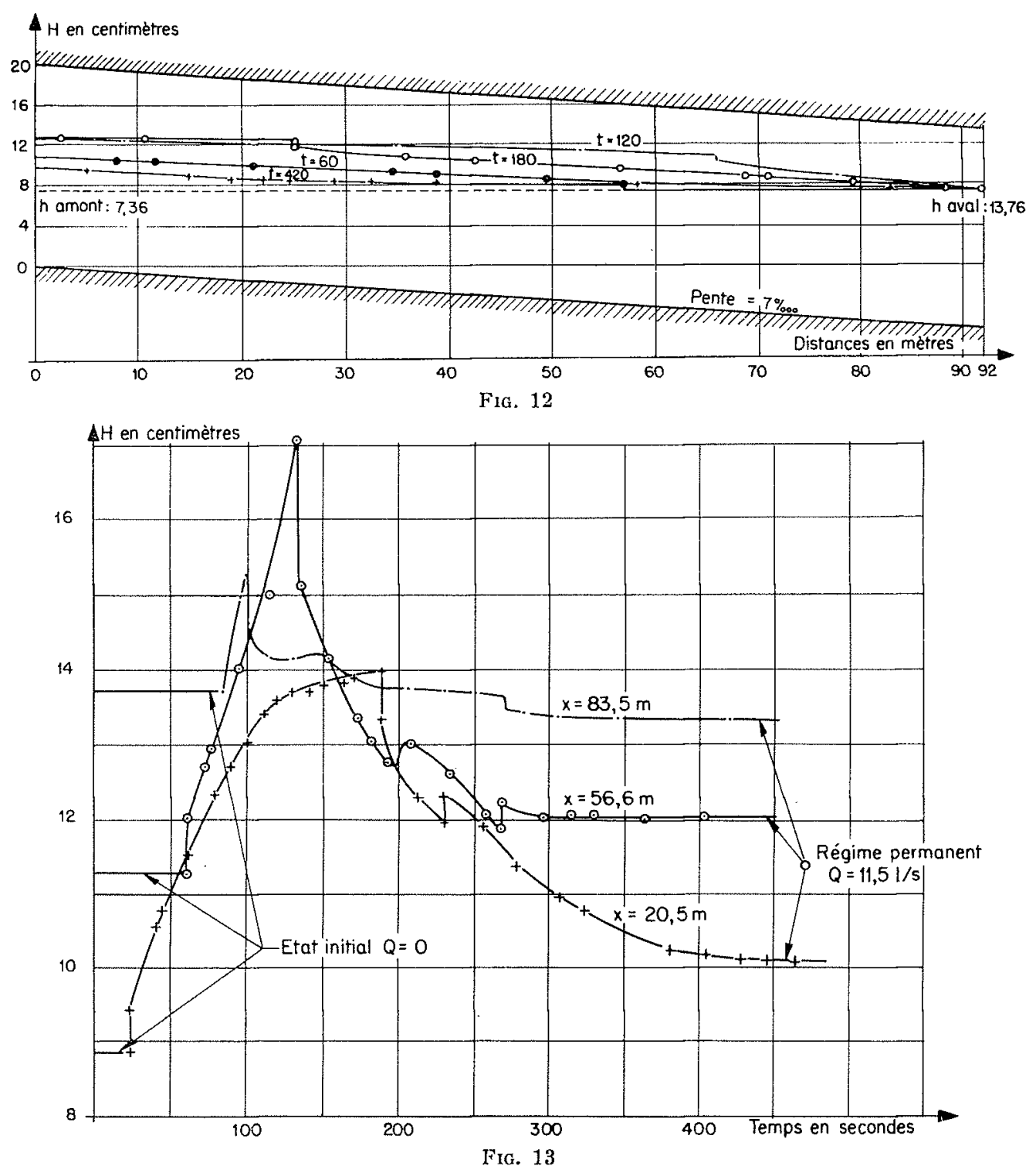

FIG. 13 


\section{CONCLUSIONS}

En conclusion, nous pouvons remarquer que les deux plus intéressantes caractéristiques de la méthode proposée sont la rapidité et la généralité.

La première qualité dépend plus particulièrement de l'instrument de calcul. Par rapport aux procédés habituels, les calculatrices digitales permettent d'employer des méthodes dont la mise en nuvre nécessite des calculs très nombreux, lonģs et compliqués. Leur rapidité de calcul autorise la répétition de nombreuses boucles d'itération qui améliorent les approximations successives d'une solution et aussi le choix d'un découpaşe du domaine d'intégration en un réseau de mailles plus fines (ce qui correspond pratiquement à augmenter le nombre $r_{0}$ de tronçons divisant le canal).

La précision offerte par l'ordinateur ne sera pas employée pleinement car, dans les problèmes intéressant l'hydraulicien, il est largement suffisant de connaître les résultats numériques à un pour cent près, car la théorie ne peut rendre exactement compte de la réalité. Mais, notons cependant que l'utilisation de huit chiffres significatifs (précision possible en langage FLEX) permet de multiplier le nombre des opérations, tout en étant assuré d'une erreur relative inférieure au centième pour le résultat final.

Le second avantage essentiel de la méthode exposée est sa grande souplesse. Nous nous sommes efforcés de donner au programme établi le maximum de généralités. Un seul programme peut, moyennant quelques modifications, permettre de traiter la majorité des cas pratiques.

Il est très facile de faire varier les données physiques ou géométriques et ainsi étudier rapidement les conséquences de variation de débit, de durée de manœuvres ou de dimensions.

L'utilisation de la fonction $\Sigma$, section mouillée, permet sans difficulté l'emploi du même programme pour différentes formes de section.

L'individualisation des calculs de $\left(\boldsymbol{A}_{0, t}\right)$ et $\left(A_{r_{o}, t}\right)$ entraîne une facilité de modification des conditions aux limites (introduction d'un déversoir, vanne de fond, etc...).

Et surtout le programme établi est valable quel que soit le type d'onde considérée, onde d'aval ou d'amont, onde positive ou négative. En outre, un calcul rapide permet de faire une étude de découpage et de la convergence de l'intégration numérique. Ceci peut être très utile dans les constructions graphiques d'intumescences et peut s'étendre d'ailleurs à la résolution de tout système d'équalions aux dérivées partielles du type hyperbolique considéré.

Cet exposé n'est qu'un bref aperçu de la méthode que nous avons mise au point et qui s'avère d'ores et déjà très fructueuse. Les résultats que nous avons obtenus nous ont encouragés à poursuivre l'exploration de multiples possibilités offertes par ce procédé de calcul d'intumescence.

\section{I S C U S I O N}

président : M Remenienas

M. le Président remercie M. Thinfió et note que la tendance actuelle est à la généralisation des méthodes de calcul à l'ordinateur électronique. D'aucuns pensent même que ce procédé supplantera à la longue le modèle I'éduil.

Le modèle réduit hydraulique classique n'est qu'un calculateur analogique établi pour résoudre un problème bien déterminé. Au contraire, les méthodes telles que celle que vient de nous exposer $M$. Thinnior peuvent s'adapter à des quantités de problèmes différents par la variation des formes analytiques et des grandeurs numériques.

M. Preissmann demande à M. Thirriot s'il a eu des intumescences de front raide (mascaret ou réseau mobile) qui constituent des discontinuités de son réseau, et comment il opère dans ce cas.

M. Thirriot explique qu'il a rencontré des intumescences à front raide dans le cas de galerie de fuite à profil circulaire, et que, pour celá, il a utilisé le programme général en introduisant la condition de front raide à l'abscisse atteinte par le front de l'intumescence, c'esta-dire qu'il a employé des formules du ressaut mobile pour déterminer la célérité. M. Thrnnió explique:

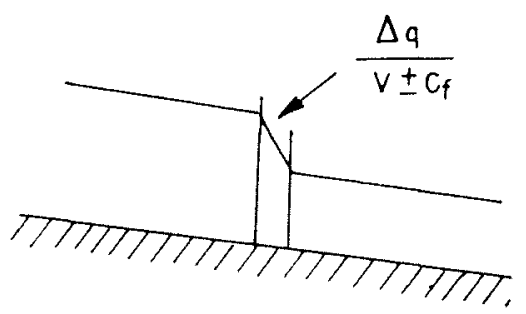

La résolution du système intégral présenté dans l'exposé se ramène à un cheminement le long d'un tronçon sur les courbes intégrales (figure). Il a donc envisagé un 
cheminement courbe correspondant aux formules différentielles jusqu'à la proximité du front d'onde où, pour l'intégration du terme $d q /(V \pm C)$ au lieu de l'intégrale, il a pris la différence finie $\Delta q /\left(V \pm \mathrm{C}_{f}\right), \mathrm{C}_{f}$ étant la célérité du front d'onde calculée d'après la formule du ressaut. Les résultats obtenus donnent quelques espérances quant à la validité de la méthode.

M. Preissmann indique qu'à la Sogreah on utilise un procédé aux différences finies qui consiste, en gros, à interpoler à chaque fois les résultats que l'on obtiendrait avec la méthode des caractéristiques. La méthode, dérivée des travaux sur la théorie des gaz et qui consiste à introduire fictivement une pseudo-viscosité, a donné des résultats très bons.

M. Preissmann dit qu'à la Sogrfah on n'a pas observé que les calculs électroniques tendraient à supprimer le modèle réduit pour un cas comme celui des ondes dans les rivières : les modèles seraient même favorisés en ce sens qu'on introduirait sur modèle certaines parties constituant des pertes de charge singulières qui sont ensuite réintroduites dans la machine mathématique. La machine remplace le modèle hydraulique le moins adéquat, ce qui est effectivement le cas pour les ondes de crue dans les rivières, en raison de la distorsion importante qu'un tel modèle impose.

M. Thingior ajoute que dans les méthodes numériques, on ne tient pas compte des petites ondulations secondaires.

M. le Président précise que, comme le rappelle M. THIRRIOT, dans le cas général, l'intumescence comporte un front d'onde qui n'est pas pris en compte dans les calculs, puisque ceux-ci négligent les accélérations verticales et les courbures; en pratique, on détermine d'abord la hauteur du corps de l'onde et ensuite, par un calcul auxiliaire, on évalue celle de l'ondulation secondaire qui est, dans certains cas, de l'ordre de la hauteur du corps (voir courbes de M. le Professeur Favre).

M. le Président remarque que lorsqu'on calcule à la machine électronique, il n'est pas évident que la transposition en calcul numérique de méthodes élégantes, telles celles que l'on emploie dans les épures de M. Craya, soit meilleure que la méthode un peu simpliste qui consiste à calculer aux différences finies; le choix doit résulter de l'estimation des prix de revient des deux modes de calcul.

M. Freissuan explique qu'il a affaire, en général, à des rivières où les variations sont relativement lentes, tandis que $M$. Thrrriot considère des rivières où les variations sont rapides, donc où la méthode des caractéristiques a ses avantages. Dans le cas des rivières, il faut des astuces d'ordre numérique de façon à accélérer le calcul qui, effectué suivant la méthode la plus simple, exigerait des temps prohibitifs.

M. le Président dit que M. Preissmann emploie les équations de Saint-Venant sous la forme initiale tandis que M. ThinRion les transforme pour les rendre accessibles au calcul graphique.

M. THIRror pense que l'intelligence dans les méthodes numériques n'intervient pas au même endroit que dans les méthode graphiques. Il y a autant d'ingéniosité dans l'organisation d'un programme et dans la réalisation et la mise au point que dans l'application des méthodes graphiques, La machine libère l'opérateur des servitudes des tracés graphiques longs et délicats el favoriser l'abstraction et la généralisation.

M. Nougaro demande à M. Preissmann si les résultats de ses études sont publiés. Il propose qu'un confrontation des méthodes numériques puisse se faire à l'occasion du prochain congrès de l'A.I.R.H. à Belgrade, cette question étant portée à l'ordre du jour.

M. Preissmann indique qu'il a fait une communication à ce sujet au Congrès de la Société Française de Calcul à Grenoble, dont le compte rendu sera publié. Il pense que M. Nougaro et lui-même pourraient présenter, même conjointement, une communication à ce sujet à Belgrade.

M. Faure demande si, dans les équations données au début de la conférence, il ne faudrait pas lire, pour le terme de frottement, $k v|v|$ au lieu de $k v^{2}$.

Réponse affirmative de M. Thirriot.

M. Faune demande quel est l'intérêt de programmer une méthode approchée dont le seul avantage est, pour certains problèmes, assez limités, de fournir la variation de hauteur dans une section sans interpolation. Il signale que sur une I.B.M. 650 ou I.B.M. 704 (programme du Laboratoire National d'Hydraulique de Chatou), un sousprogramme permet d'obtenir l'interpolation.

Aux objections de M. Faure, M. Thirhot répond que :

a) L'interpolation est un procédé numérique éprouvé et qui ne présente pas de difficulté de programmation. Mais l'introduction d'une interpolation alourdit le temps de calcul, surtout dans le cas d'une machine I.B.M. 650 . Il vaut mieux effectuer manuellement les interpolations nécessaires, linéaires ou paraboliques, lors du déponiliement. Celui-ci est d'ailleurs assez rapide.

b) Il n'est pas besoin de programmer une méthode approchée, car elle n'est qu'un cas particulier de la méthode générale. Le choix du découpage et quelques shunts judicieux permettent d'utiliser le programme général dans l'hypothèse simple de fixité des profils de calculs.

M. Ie Président signale que, généralement, le temps nécessaire à la machine pour exécuter les calculs est très faible; par contre, la préparation des programmes est parfois longue et onéreuse. Aussi, l'habitude s'estclle instaurée, dans certains domaines, de vendre ou de prêter les programmes, afin d'éviter un gaspillage intellectuel et matériel.

M. le Président remercie encore une fois les auteurs de la communication et, en particulier, M. THirrior qui l'a exposée. 